\title{
Lung structure recognition: a further study of thoracic organ recognitions based on CT images
}

\author{
X. Zhou ${ }^{\text {a }}$, S. Kobayashi ${ }^{\text {a }}$, T. Hayashi ${ }^{\text {a }}$, N. Murata ${ }^{\text {a }}$, T. Hara ${ }^{\text {a }}$, H. Fujita ${ }^{\text {a }}$, \\ R. Yokoyama ${ }^{\mathrm{b}}$, T. Kiryu ${ }^{\mathrm{b}}$, H. Hoshi $^{\mathrm{b}}$, M. Sato ${ }^{\mathrm{c}}$ \\ ${ }^{a}$ Dept. of Intelligent Image Information, Division of Regeneration and Advanced Medical \\ Science, Graduate School of Medicine, Gifu University, Japan \\ ${ }^{\mathrm{b}}$ Dept. of Radiology, Gifu University School of Medicine \& University Hospital, Gifu \\ University, Japan \\ ${ }^{c}$ Dept. of Image Engineering, Tokyo Institute of Polytechnics, Japan \\ Email (Corresponding Author, X. Zhou): zxr@vsl.gifu-u.ac.jp
}

\begin{abstract}
We are developing a Computer Aided Diagnosis (CAD) System for extracting and recognizing thoracic organ regions from chest $\mathrm{CT}$ images. In a previous study, we have shown that this system can automatically recognize 9 kinds of human organ and tissue regions from multi-slice CT images and provide some useful applications for visualizing CT images three-dimensionally (3-D) or two-dimensionally (2-D) based on preliminary recognition results. In this paper, we propose some further studies for this system that focus on lung structure recognition. These studies include: (1) Identification of thoracic cage region, (2) Extraction of major and minor fissures and classification of lung regions, (3) Classification of lung surface and identification of hilus pulmonis. The proposed methods have been applied to three chest CT images for recognizing lung structure and have demonstrated promising results.
\end{abstract}

Keywords: 3-D CT image, organ region segmentation, lung structure recognition

\section{Introduction}

The remarkable progresses of multi-slice CT technology enable radiologists to scan a larger volume of human body in a shorter scan time with a higher isotropic spatial resolution in onetime CT photography. Now, the newest CT scanner can easily provide a high quality (spatial resolution about $0.63 \mathrm{~mm}$ ) CT image covering the whole human chest within one breath hold time. However, radiologists have to spend much time and energy to view a huge number of individual images slice by slice on screen for each patient case. Therefore, computer-aided diagnosis (CAD) and visualization of 3-D CT images are strongly desired to reduce this burden.

A CAD system always needs the following functions: (1) detecting suspicious regions (SR) in different organs and giving a discrimination of benignancy or malignancy for each SR, (2) visualizing interesting regions of a CT image in 2-D or 3-D. For each function of CAD 
system, recognizing the different organ and tissue regions and understanding the normal structure of human body from a CT image are necessary. We have been developing a system for extracting and recognizing thoracic organ and tissue regions automatically from 3-D multi-slice CT images [1,2]. We have presented the processing procedures used in this system for recognizing 9 kinds of internal organ and tissue regions and showed preliminary experimental results [1].

In this paper, we present some recent research works for this system, which centre on automated recognition of lung structure from CT images. These works include three parts: (1) automated identification of thoracic cage, (2) automated extraction of major and minor fissures and classification of lung regions, (3) automated classification of lung surface and identification of hilus pulmonis. In section 2, we describe the processing methods of each part, and then, those methods are applied to real chest $\mathrm{CT}$ images and recognition results are shown in section 3 with a brief discussion.

\section{Methods}

The scheme for recognizing lung structure has five processing steps: (1) Extracting thoracic cage region and separating lung from the other regions, (2) Extracting trachea and bronchus and recognizing bronchial tree, (3) Extracting major and minor fissures and dividing lung into five lobe regions, (4) Identifying hilus pulmonis from lung surface and recognizing artery and vein on the sectional surface of hilus pulmonis, (5) Extracting lung vessels and recognizing branches of pulmonary artery and vein. Steps 1-4 are described in the following along with the middle result images in experiments shown in section 3.

\subsection{Automated identification of thoracic cage region [3]}

The processing flow for identifying thoracic cage region is showed in Fig.1(a). We used a grey scale thresholding and a 3-D region growing method to extract the skeleton region[Fig.2(a)] from original CT images firstly, and then, a classification has been applied to separate the skeleton region into six parts (Spinal column, Rib, Cartilage, Sternum, Collarbone, Shoulder blade) using their shape, location and density features [Fig.2(b)]. An initial boundary of thoracic cage [Fig.2(c)] is created through stretching the inscribed surface of Spinal column, Rib, Cartilage and Sternum. Last, an active contour model (ACM) is applied to generate the final boundary of thoracic cage by moving the sample points (that are selected at equal intervals along the initial boundary slice-by-slice) and fitting them to the contour of the thoracic cage [Fig.2(d,e)]. Using this boundary, we can separate the internal organ regions from muscle, skeleton and fat regions of the human body.

\subsection{Automated extraction of lung lobe regions and fissures from lungs [4]}

Fig.1(b) shows the processing flow of lobe region segmentations. The lung regions are extracted using a gray scale thresholding and a connected component processing firstly [Fig.3(d)]. And then, the airway of bronchus is extracted using a 3-D region growing method [Fig3(a)]. A 3-D axis-thinning algorithm is used to extract the bronchial tree [4] firstly, then, 


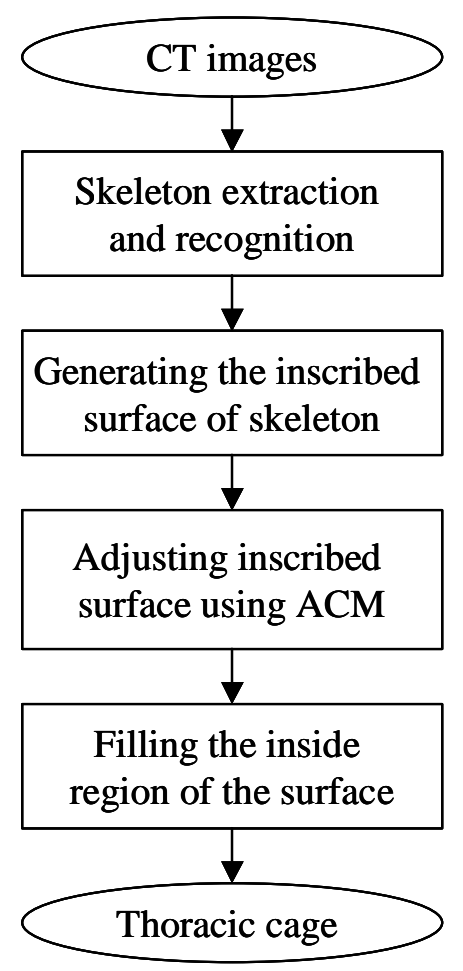

(a) Extraction of thoracic cage

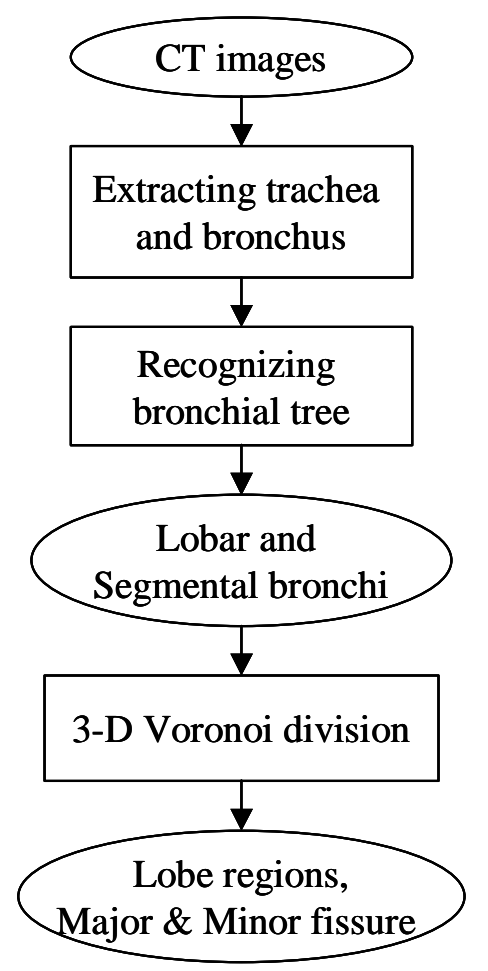

(b) Extraction of lobar and fissure

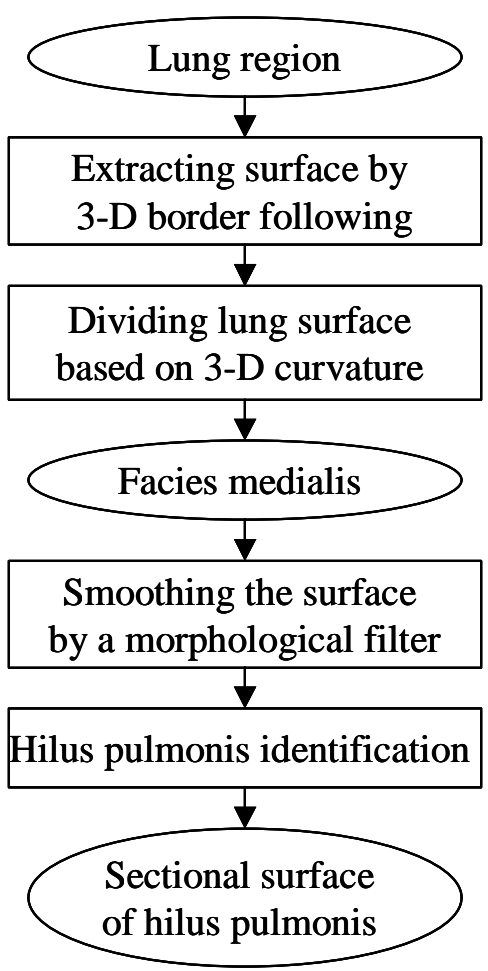

(c) Identification of hilus pulmonis

Fig.1: Processing flows for lung structure recognition.

an anatomy knowledge based marching method is used to recognize bronchial tree structure [Fig.3(b)]. Using the distance to each segmental bronchus in left and right lung regions [Fig.3(c)], Lung regions are divided into five lobes by a 3-D Voronoi division method [5]. The contact surfaces between different lobes are regarded as major or minor fissures [Fig.3(e)].

\subsection{Automated classification of lung surface and identification hilus pulmonis [6]}

A processing flow is developed to extract sectional surface of hilus pulmonis [Fig.1(c)]. This flow extracts the surface of lung regions using a 3-D border following algorithm at first, and then, divides the left and right lung surface into facies costalis, facies diaphragmatica and facies medialis [Fig.4(a,b,c)] based on 3-D curvature features. We use a morphological filter to create a smooth curve to fit facies medialis. Through analyzing the differentiation of smooth curve and facies medialis, we can decide the position of hilus pulmonis and get a sectional surface of it [Fig.4(d)]. We made a 3-D view of lung vessels from the sectional surface [Fig.4(e)] and found that it is possible to distinguish the positions of artery and vein on this surface based on anatomy knowledge. 


\section{Results}

We applied the proposed methods to three patient cases of multi-slice CT images. Each image covers the entire human chest region with isotropic spatial resolution of about $0.63 \mathrm{~mm}$ and 12-bit density resolution. We show some experimental results in Fig.2-4, and can conclude the following: (1) The methods proposed in section 2 can extract target regions successfully. It appears that our scheme is proper for lung region classification. (2) The recognition results are very useful for CT image analysis. Based on the recognition results, we can easily view the internal organs in 3-D and solve the overlap problem caused by skeleton, muscle and fat regions out of thoracic cage. After lung region classification, we can view lung vessels and branches locating at each lobe region and make a further analysis. The hilus pulmonis extraction is helpful for analyzing the structure of mediastinum, which is very important for pulmonary artery and vein classification.

\section{Conclusions}

We have been developing a system for automated recognition of human organ and tissue regions from CT images. In this paper, we proposed three methods used in this system for extracting thoracic cage, sectional surface of hilus pulmonis and lobe regions of lung. We confirmed that these methods are effective using experimental results.

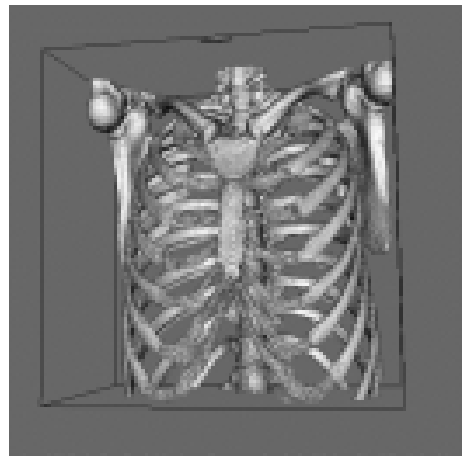

(a) Skeleton region

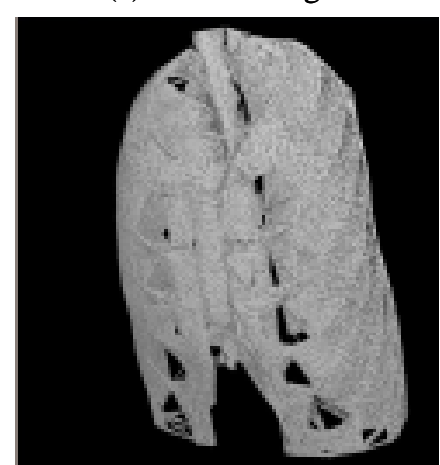

(c) Initial boundary of thoracic cage

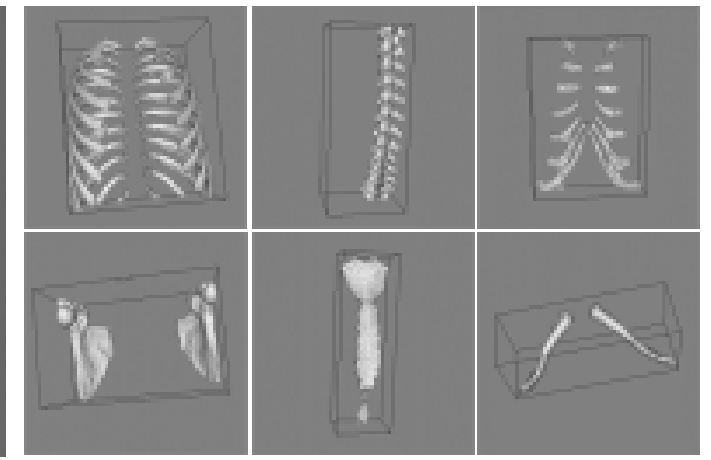

(b) Classification results of skeleton

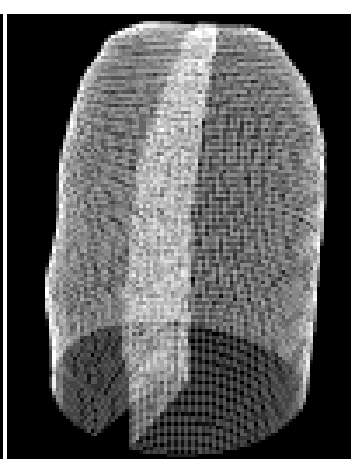

(d) Boundary of thoracic cage before ACM

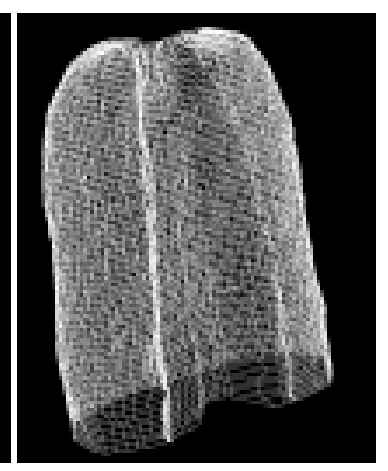

(e) Boundary of thoracic cage after ACM

Fig.2: Result images of thoracic cage extraction. 


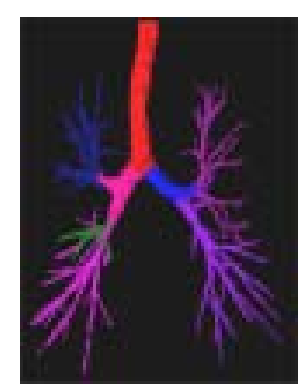

(a) Bronchus

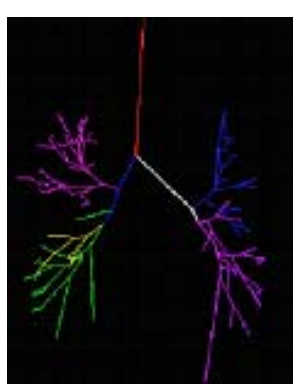

(b) Bronchial tree

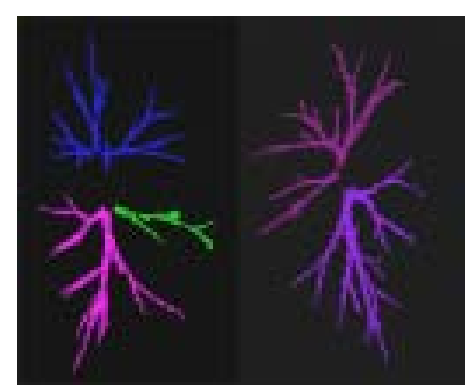

(c) Segmental bronchi(right, left)

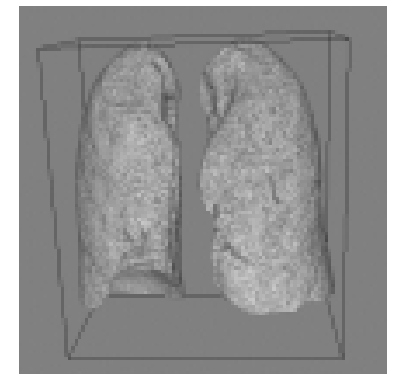

(d) Lung regions

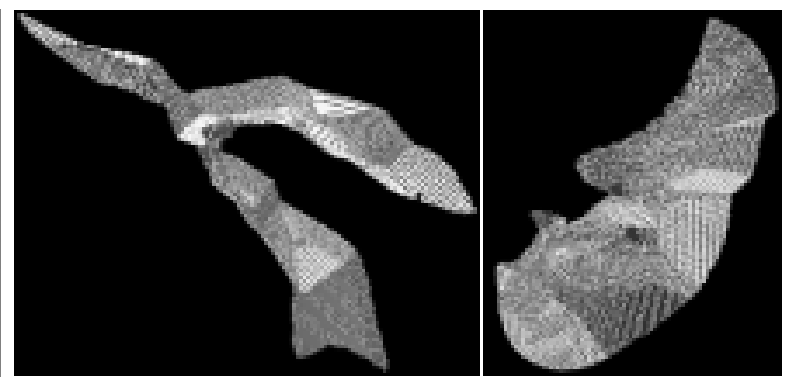

(e) Fissures in right and left lung

Fig.3: Result images of lung lobe regions and fissures extraction.

\section{Acknowledgements}

Authors thank the members of Fujita's Laboratory and Virtual System Laboratory (VSL) of Gifu University for their collaboration. This research was supported in part by research grants from the Collaborative Centre for Academy/Industry/Government and VSL of Gifu University, in part by the Ministry of Health, Labour, and Welfare under a Grant-In-Aid for Cancer Research and in part by the Ministry of Education, Culture, Sports, Science and Technology under a Grant-In-Aid for Scientific Research, Japanese Government.

\section{References}

[1] X. Zhou, T. Hara, H. Fujita, Y. Ida, K. Katada, K. Matsumoto: "Extraction and Recognition of the Thoracic Organs Based on 3D CT Images and Its Application", Proc. of the 16th International Congress and Exhibition of Computer Assisted Radiology and Surgery 2002, pp.776-781, 2002.

[2] X. Zhou, T. Hara, H. Fujita, R. Yokoyama, T. Kiryu, H. Hoshi, M. Sato: "Preliminary Examinations of Automated Tissues and Organs Recognition from Multi-slice Torso CT Images", Journal of Medical Imaging and Information Scienceces, Vol.20, No.1, pp.44-47, 2003. (In Japanese)

[3] S. Kobayashi, X. Zhou, T. Hara, H. Fujita, Y. Ida, K. Katada, K. Matsumoto: ” Extraction of Thoracic Cage Based on Bone Structure from 3D Chest CT Images", Technical Report of IEICE, MI2002-53, pp. 35-39, 2002. (In Japanese) 


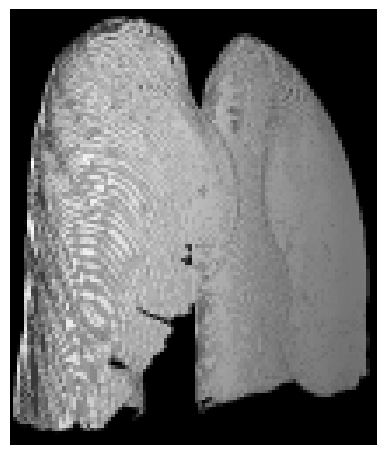

(a) Facies costalis

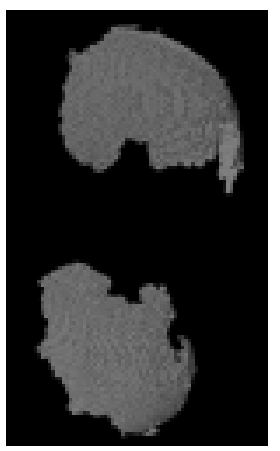

(b) Facies diaphragmatica

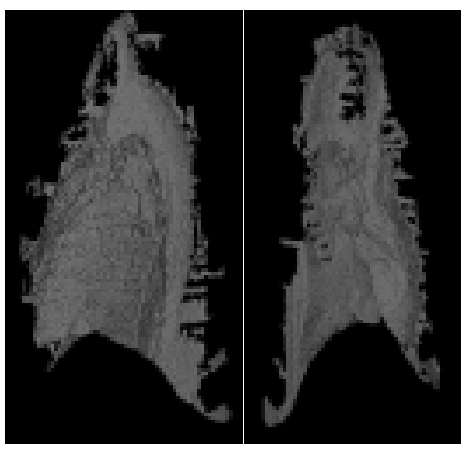

(c) facies medialis

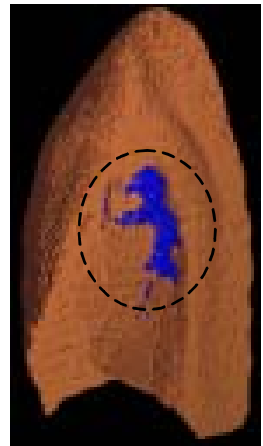

(d) Sectional surface of hilus pulmonis

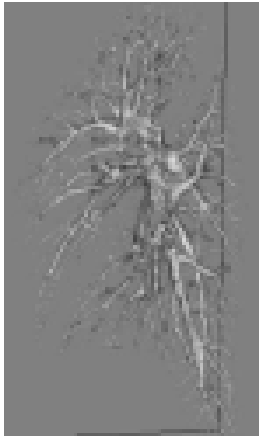

(e) Lung vessels from hilus pulmonis

Fig.4: Result images of lung surface classification and hilus pulmonis extraction.

[4] T. Hayashi, X. Zhou, T. Hara, H. Fujita, Y. Ida, K. Katada, K. Matsumoto: " Primary Investigation on Classification of Lung Field based on Bronchus Using Chest Multi-slice CT Images", Technical Report of IEICE, MI2002-79, pp. 57-63, 2002. (In Japanese)

[5] T. Saito, J. Toriwaki: "Algorithm of Three Dimensional Euclidean Distance Transformation and Extended Digital Voronoi Diagram, and Analysiss of Human Liver Section Images", Journal of the Institute of Image Electronics Engineering of Japan, Vol.21, No5, pp.468-474, 1992. (In Japanese)

[6] N. Murata, X. Zhou, T. Hara, H. Fujita, R. Yokoyama, T. Kiryu, H. Hoshi, M. Sato: ” Automated Extraction of Hilus Pulmonis from Multi-slice Chest CT Image", Technical report of IEICE, MI2002-101, pp.31-35, 2003. (In Japanese) 